\title{
Deutsch als Ausgangssprache für die Erneuerungsbewegung der wirtschaftlich-juristischen Fachsprache in Ungarn ${ }^{1}$
}

\author{
Beáta SZÉP
}

\begin{abstract}
German as a source language in the emergence and development of economic and legal terminology in Hungary

The study presented in this paper reviews the emergence and development of Hungarian economic and legal terminology. It is based on research during which I examined over 1000 terms from their first publication in specialized dictionaries, through changes in their meaning, all the way to their integration into technical vocabulary or their extinction. My research also focused on the reasons why the colloquial meaning of a term may cause the disappearance of its technical meaning. I conclude my study with examples.
\end{abstract}

Keywords: terminology, diachronic research, legal vocabulary, technical meanings

DOI: doi.org/10.15452/StudiaGermanistica.2021.28.0007

\section{Einführung}

In diesem Beitrag werden in erster Linie die Herausbildungs- und Entwicklungsprozesse der ungarischen wirtschaftlich-juristischen Fachsprache dargestellt. In meiner Forschung habe ich mehr als eintausend Termini analysiert von ihrem ersten Erscheinen in einem Fachwörterbuch durch ihren Bedeutungswandel bis zum Etablieren in dem oder zum Aussterben aus dem Fachwortschatz. Als Schluss wird dafür ein konkretes Beispiel dargestellt.

Die Herausbildung der Fachsprachen ist in der Geschichte aller Sprachen ein langer Prozess, der sich manchmal sogar über Jahrhunderte hinaus vollzieht. Die Wörterbücher aus den 17.-18. Jh. bezeugen schon vereinzelte Versuche nicht nur in Ungarn, sondern auch in mehreren mitteleuropäischen Ländern. Man kann aber von bewusst gesteuerten und organisierten Arbeiten an der Sprachentwicklung erst seit dem 19. Jh., besonders seit der zweiten Hälfte des 19. Jhs. sprechen, als die Ergebnisse der Debatten über die Spracherneuerung es schon ermöglicht haben, auch auf die

\footnotetext{
1 Die Forschung wurde im Rahmen des Projektes EFOP-3.6.1-16-2016-00001 „Komplexe Entwicklung der Forschungskapazitäten und Dienstleistungen an der Eszterházy Károly Universität“" gefördert.
} 
einzelnen Detailfragen Acht zu geben. Eine solche Frage war die muttersprachliche Bestimmung (d. h. Benennung) von allgemeinen Begriffen des Rechtswesens und der Verwaltung.

Die Entwicklung der juristischen Fachsprache hängt natürlich mit der Geschichte der Kodifikation des Rechts eng zusammen. Eine Kodifikation ist die systematische Zusammenfassung der Rechtssätze eines Rechtsgebiets in einem einheitlichen Gesetzeswerk, in dem das jeweilige Rechtsgebiet abschließend geregelt werden soll. Ein klassischer Vertreter der Kodifikation ist z. B. das deutsche Bürgerliche Gesetzbuch (BGB).

Die Geschichte der Kodifikation des ungarischen privaten Rechts kann in die folgenden Epochen aufgeteilt werden:

1. Periode von der Arpadenzeit bis 1486 (Veröffentlichung des Decretum maius, Gesetzbuchs von König Matthias)

2. Periode von 1486-tól bis zum Landtag von 1832/36 (Anfang der Reformzeit)

3. Periode von dem Anfang der Reformzeit bis 1900

4. Periode von dem ersten Entwurf bis die sozialistische Kodifikation (1959)

5. Periode von 1959 (sog. ,,altes“ Ungarisches Bürgerliches Gesetzbuch) bis 2013 (Veröffentlichung des ,neuen“ ungarischen BGBs)

6. Periode seit 2013

In diesem Beitrag wird in erster Linie auf die ungarische Erneuerungsbewegung der wirtschaftlichjuristischen Fachsprache fokussiert. Dieser Prozess vollzog sich hauptsächlich in der zweiten und dritten Periode der obigen Gliederung, aber besonders die gesellschaftlich-historischen Ereignisse und gesetzliche Entwicklungsprozesse der Reformzeit sind für unsere Forschung relevant. Während die zweite Periode durch vereinzelte sprachliche Erneuerungen geprägt war, herrschte in der dritten schon das bewusste, kollektive Sammeln von Termini verschiedener Fachbereiche, dessen Repräsentanten die juristisch-politische Wörterbücher sind. Diese Periode gilt als die Epoche der bewussten Fachspracherneuerungen nicht nur in Ungarn, sondern im ganzen Mitteleuropa.

Die Juristen verlangten in dieser Epoche schon wahre Reformen. Die Idee der nötigen Kodifikation wurde von Ferenc Deák am Landtag von 1832/36, genauer an der Sitzung am 25ten Mai 1834 ins Leben gerufen. Die damalige wirtschaftliche Lage des Landes benötigte die Umstrukturierung der alten bürgerlichen Ordnung. Graf István Széchenyi war der Erste, der seine Zeitgenossen darauf aufmerksam machte. Deák betonte in seiner Rede die Unklarheit der geltenden Gesetze.

Die Kodifizierungsarbeiten resultierten zwischen 1836 und 1847 zuerst im Bereich der Wechsel- und Handelsrecht zahlreiche Gesetze, die sog. excerpták (Exzerpten, d.h. ,Auszüge'), die pure Rezeptionen der deutschsprachigen Gesetze waren. Die konkreten Beispiele dafür werden unter ausführlicher behandelt.

Der Wortschatz der ungarischen juristischen Fachsprache entstand also als Ergebnis der deutschungarischen Fachübersetzung.

$\mathrm{Zu}$ dieser Kodifizierungsarbeit benötigte man einen ungarischen Fachwortschatz, deshalb waren zahlreiche Wörterbücher publiziert. Laut dem Gesetz von 1836 über die ungarische Sprache war das Königliche Oberlandesgericht auch schon verpflichtet, in den ungarischen Prozessen in ungarischer Sprache zu urteilen. Nach den Gesetzen von 1840 und 1843/44 wurde das Ungarische zur offiziellen Sprache in Ungarn.

All dies führte zur Veröffentlichung weiterer Wörterbücher, die die Ergebnisse der fachsprachlichen Übersetzung darstellen. Dieser Prozess wird im Folgenden dargestellt.

\section{Die erste Epoche - Vereinzelte Veröffentlichungen}

Bis zum 16. Jh. konnte von ungarischer juristischer Fachsprache nicht gesprochen werden: In den lateinsprachigen Schriften sind ungarische Termini nur sporadisch zu finden. Als Schöpfer der ungarischen juristischen Fachsprache wird die Generation von Tamás Nádasdy, also die sog. 
Nádasdy-Garde betrachtet. Nach der Schlacht bei Mohács begann diese Generation zuerst in ungarischer Sprache zu schreiben. In der Periode danach, also in den 17-18. Jh. ist keine bedeutende Entwicklung der ungarischen juristischen Fachsprache zu beobachten. Aber auch aus dieser Periode ist schon ein Werk erwähnenswert: die erste ungarisch-lateinische Auflage vom Werböczys Decretum (Werbőczy 1611), in dessen Anhang sich ein umfangreiches ungarisch-lateinisches Fachwörterbuch namens „Index verborum Latino-Hungaricus“ befindet. Der Index wurde später auch von József Ponori Thewrewk herausgegeben (Ponori Thewrewk 1844).

Die Bedingungen sowohl für die Erneuerung der ungarischen Sprache als auch für die Schöpfung und Entwicklung der juristischen Fachsprache - und damit für die der Fachwörterbücher - wurden also einerseits durch die politischen Maßnahmen und die Gesetze seit Ende des 18. Jahrhunderts, andererseits durch die rasante wissenschaftliche Entwicklung des 19. Jahrhunderts geschaffen. Zur Zeit dieser Maßnahmen wurden immer mehr juristische Publikationen in ungarischer Sprache veröffentlicht. Als die gesetzlichen Maßnahmen den Gebrauch der ungarischen Sprache also auch im Rechtsleben ermöglichten, ist schon der dazu erforderliche Wortschatz durch die Wissenschaft - in den vielen Publikationen am Jahrhundertanfang und aufgrund deren in den Fachwörterbüchern - geschaffen worden, sogar viel mehr als es nötig gewesen wäre: Die ersten Fachwörterbücher waren in Wirklichkeit eher nur Wortsammlungen mit Riesenmengen von Synonymen. Der Grund ist am Mangel der Zentralisierung zu finden: Die verschiedenen Autoren in Budapest, Kaschau, Veszprém usw. vermuteten nicht, dass sie vereinzelt dasselbe Ziel erreichen wollten. Diese Autoren verfügten über keine Anleitungen und manchmal auch über keine sprachwissenschaftliche Bildung, trotzdem sind ihre Werke von großer Bedeutung, da sie den Bedarf vorläufig befriedigt haben, denn man kann in dieser Periode fast ein Jahrzehnt lang (von 1807 bis 1815) kein neues Produkt finden (vgl. Bakos 1880).

\section{Bewusste Fachspracherneuerungsbewegung durch Übersetzung}

Infolge der rechtswissenschaftlichen Entwicklung in Ungarn wurde auch die Vermehrung des ungarischen juristischen Fachwortschatzes notwendig. Das war der eine Grund für die Veröffentlichung von den zahlreichen juristischen Sachwörterbüchern - als Ergebnisse von Fachübersetzungen - und sonstigen Hilfsbüchern, die schon kompletter und gründlicher als ihre Vorgänger waren. Durch die enorm vielen Werke wurde aber die Entwicklung der ungarischen juristischen Fachsprache in gewisser Hinsicht eher gehemmt: einerseits wurden die Neuschöpfungen nicht definiert, was das Zurechtfinden unter den zahlreichen Synonymen erschwerte, andererseits passte sich die Mehrheit dieser Neuschöpfungen nicht in das System der ungarischen Sprache. Sie wirkten als fremd, was die Linguisten sofort kritisierten. Manche Werke waren sogar dadurch gekennzeichnet, dass die Fremdwörter nicht nur ein, sondern manchmal sogar 3, 4 oder mehr ungarische Äquivalente hatten (vgl. Terestyéni 1955).

Die verschiedenen Komitate führten inzwischen in allen Zweigen des offiziellen Rechtslebens den ungarischen Sprachgebrauch ein. Die wichtigste Epoche für die Schöpfung der Fachwörterbücher war also die Periode zwischen dem Landestag von 1832-36 und die Jahrhundertwende. Diese nationale Fachspracherneuerungsbewegung entfaltete sich infolge der globalgesellschaftlichen Änderungen im Geistesleben und sogar in der ganzen Zivilisation, und sie schloss sich den organisierten und geleiteten Spracherneuerungen im Mitteleuropa von der zweiten Hälfte des 18. Jhs. bis das dritte Drittel des 19. Jhs. an (vgl. Nyomárkay 2007).

\section{Das Deutsche als Ausgangssprache der ungarischen Fachsprachenent- wicklung}

Die bedeutende Rolle der deutschen Fachsprache in der ungarischen Kodifizierung wird eindeutig, wenn wir die oben schon erwähnten sog. excerpták (Exzerpten, d. h. ,Auszüge') unter die Lupe nehmen. Diese Exzerpte sind in erster Linie in den Gebieten des Wechsel- und Handelsrecht verabschiedet worden. 
Auf die Entwicklung des Wechselrechts Ungarns hatte das österreichische Wechselrecht eine außenordentliche Wirkung: Mit der Arbeit an dem ersten Wechselgesetz - dem sog. alten Wechselgesetz - Ungarns wurde eine Kommission unter der Leitung von Ferenc Deák beauftragt. In diese Kommission ,hat die königliche Hofkanzlei auf die Inspiration des Kanzlers Herzog Metternich als Beirat Ignaz Wildner von Maithensen den Wechsel- und Handelsjuristen von Wien in die Kommission delegiert" (Pókecz Kovács 2011:117-118). Wildner hat nach seinem Jurastudium an der Wiener Universität bei der kaiserlichen und königlichen Kammer gearbeitet, später unterrichtete er als Professor der Universität in Wien, daneben war er auch als Hof- und Gerichtsrechtsanwalt tätig. Ferencz Pulszky, der als Zeichner an der Arbeit der Kommission teilgenommen hat, schrieb in seinem Erinnerungsband darüber folgendes (Pulszky 1884): Wildner hat das Gesetz auf Deutsch zusammengestellt, und dies bedeutete eher nur eine mechanische Arbeit, da ein Wechselgesetzbuch im Wesentlichen überall das gleiche, also international ist. Wildners Entwurf diente als Grundlage der Arbeit der Kommission, und sie haben daran kaum etwas geändert: Das erste ungarische Wechselgesetz ist das Ergebnis der Fachübersetzung, d.h. diese Arbeit bedeutete auch die Übersetzung der deutschen wirtschaftlich-juristischen Termini ins Ungarische! Und nicht nur diese: Auch zahlreiche andere Gesetze des ungarischen Wirtschaftslebens entstanden ebenso durch - teilweise deutsch-, teilweise französisch-ungarische - Übersetzung, wie z. B. das Konkursgesetz oder die Handelsgesetze (vgl.: Pókecz Kovács 2011).

Diese Übersetzungsprozesse resultierten dann in viele deutsch-ungarische Fachwörterbücher, durch deren Untersuchung wir eine detailliertere Übersicht der Entwicklung der wirtschaftlich-juristischen Fachsprache geben können. In meiner Forschung fokussierte ich mich auf den größten Versuch in diesem Bereich: ,Das Törvénytudományi Müszótár' (Das Gesetzwissenschaftliche Sachwörterbuch), das zuerst 1843 und später 1847 (zweite, erweiterte Auflage) herausgegeben wurde. Wie es in der Vorrede des Editors Ferencz Schedel steht, habe man mit den Vorbereitungen vom ,Magyar Nagy Szótár' (Ungarischen Großwörterbuch) laut dem Beschluss der ersten Tagung von Magyar Tudós Társaság (der Ungarischen Gelehrtengesellschaft) (1831) angefangen. Die erste Phase dieser Arbeit war das Sammeln von Termini: Zuerst versuchte man die bisher publizierten Werke aufzulisten. Danach wurden diese Werke an die Mitarbeiter des Wörterbuchs verteilt, die dann daraus alle Termini sammeln sollten (Schedel 1847). So wurden zuerst das ,Mathematische“ und ,Philosophische“, dann als drittes das ,Gesetzwissenschaftliche Sachwörterbuch` herausgegeben. Diese Epoche spiegelt sich in der Zielsetzung der Autoren gut wider: Die gesammelten Sachwörter seien keine zu akzeptierenden Vorschläge der Gesellschaft (Schedel 1847). Mit dem Sachwörterbuch wollten sie die Lücken aufdecken und die aufgefundenen Termini nun überschaubar und vergleichbar machen, damit man statt der vorhandenen keine neuen schafft (Schedel 1847). Waren die Wortsammlungen früherer Zeiten also durch die individuellen Schöpfungen und das vereinzelte Sammeln geprägt, so wollte man hier mit der Veröffentlichung des Wörterbuchs die Fachspracherneuerungsarbeit schon bewusst auf einen abgestimmten Weg treiben. Das wurde auch im Vorwort des Wörterbuchs betont: Aus diesen Vormaßnahmen könne das Publikum entnehmen, dass die Gesellschaft nicht jegliche Sachwörter gleich akzeptiere, sondern nur diejenigen ins Großwörterbuch aufnehme, die sich dem Sprachsystem anpassen und den Begriff sinnvoll ausdrücken könnten; weiterhin, dass die Gesellschaft keinesfalls stur und willkürlich, sondern aufgrund gemeinsamer Vereinbarung verfahren wolle (Schedel 1847).

\section{Lehnübersetzung als wichtige Methode der Fachspracherneuerung}

Neben Wortbildung und Neuschaffungen war die Lehnübersetzung auch in der fachsprachlichen Erneuerung der ungarischen Sprache im 19ten Jahrhundert die meistbenutzte Methode. Am häufigsten kommen die zusammengesetzten Wörter (Stand $\mid$ punkt > állás $\mid$ pont) oder die Verben/ Nomina mit Vorsilben (aus $\mid$ stellen > ki $\mid$ állit; Ein $\mid$ fluss > be $\mid$ folyás) als Lehnübersetzungen vor, aber auch Wortbildungen wurden auf diese Weise übersetzt (Keller/Kellner > pince/pincér).

Nyomárkay $(2004 ; 2010)$ weist in seinen kontrastiven Analysen zwischen den kroatischen und ungarischen Lehnübersetzungen darauf hin, dass es im Ungarischen viel mehr zusammengesetzte 
Wörter gibt und diese genau die deutschen Muster nachahmen. Es wurde auch bewiesen, dass viele Wörter im Kroatischen, nicht direkt aus dem Deutschen kamen - wie es früher behauptet wurde -, sondern eindeutig die ungarische übergebende Rolle aufweisen.

Die deutsche Wirkung fällt auch im Fall der phonologischen Adaptation auf. Im Kreis der Termini war dies eine seltener angewandte Methode, und diese Fachwörter waren auch nicht lang Elemente des ungarischen Fachwortschatzes. In meinem Forschungsmaterial von 1050 Termini sind nur sehr wenige Beispiele dafür zu finden, wie z. B. börze $(<$ Börse), die heute in der ursprünglichen fachsprachlichen Bedeutung nicht mehr existiert, oder die Vorsilbe von fár $\mid$ dijj $(<$ Fahr $\mid$ gebühr $)$, die sich nicht ins Ungarische eingewurzelt hat.

\begin{tabular}{|c|c|c|}
\hline Deutsch & Ungarisch & Heutige Form \\
\hline $\begin{array}{l}- \text { frei } \\
\text { steuerfrei } \\
\text { gebührenfrei }\end{array}$ & $\begin{array}{l}\text {-ment }(1808) /- \text { mentes }(1835) \\
\text { adóment/adómentes } \\
\text { dijment/dijmentes }\end{array}$ & $\begin{array}{l}\text { mentes } \\
\text { adómentes } \\
\text { díjmentes }\end{array}$ \\
\hline $\begin{array}{l}\text { unterschreiben } \\
\text { Unterschreibung } \\
\text { Unterschrift }\end{array}$ & $\begin{array}{l}\text { alája ír }(1808) \\
\text { aláirás }(1803) \\
\text { alólirás }(1803)\end{array}$ & $\begin{array}{l}\text { aláir } \\
\text { aláirás }\end{array}$ \\
\hline $\begin{array}{l}\text { Grund } \\
\text { Grundregel } \\
\text { Grundsatz } \\
\text { gründen }\end{array}$ & $\begin{array}{l}\text { alap }(1774) \\
\text { alapszabály }(1838) \\
\text { alapelv }(1834) \\
\text { alapit }(1792)\end{array}$ & \begin{tabular}{|l|} 
alap \\
alapszabály \\
alapelv \\
alapit
\end{tabular} \\
\hline $\begin{array}{l}\text { Versteigerung } \\
\text { versteigern }\end{array}$ & $\begin{array}{l}\text { árfelverés (1803) } \\
\text { árper / kótyavetye (1807) } \\
\text { árperel / kótyavetyél (1807) } \\
\text { elárverez (1845) }\end{array}$ & $\begin{array}{l}\text { árverés } \\
\text { elárverez }\end{array}$ \\
\hline Wertstand / Kurs & $\begin{array}{l}\text { pénzbecsfolyamat }(1803) \\
\text { pénzfolyamat }(1823) \\
\text { árfolyam }(1832) \\
\text { pénzkelet / pénzbecs }(1848) \\
\text { árkelet }(1858)\end{array}$ & árfolyam \\
\hline $\begin{array}{l}\text { Artikel } \\
\text { Warenartikel }\end{array}$ & $\begin{array}{l}\text { czikk }(1786) \\
\text { áruczikk }(1835)\end{array}$ & \begin{tabular}{|l|l} 
cikk \\
árucikk
\end{tabular} \\
\hline Geschäft & $\begin{array}{l}\ddot{u g y}(1838) \\
\text { ügyelet }(1831) \\
\text { ügylet }(1845) \\
\text { üzlet }(1841) \\
\end{array}$ & \begin{tabular}{|l}
$\ddot{\text { ügy }}$ \\
*ügyelet ('Dienstbereitschaft') \\
ügylet \\
üzlet \\
\end{tabular} \\
\hline $\begin{array}{l}\text { vereinigen } \\
\text { Vereinigung } \\
\text { Verein } \\
\end{array}$ & $\begin{array}{l}\text { együl (1836) } \\
\text { együlés (1836) } \\
\text { egyület (1836) / egyesület (1815) / egylet (1845) } \\
\end{array}$ & \begin{tabular}{|l|} 
egyesül \\
egyesülés \\
egyesület
\end{tabular} \\
\hline $\begin{array}{l}\text { Fabrik } \\
\text { (fabrikmäßig) erzeugen }\end{array}$ & $\begin{array}{l}\text { gyár }(1830) \\
\text { gyárt }(1835)\end{array}$ & $\begin{array}{l}\text { gyár } \\
\text { gyárt }\end{array}$ \\
\hline $\begin{array}{l}\text { Industrie } \\
\text { Industrieller }\end{array}$ & $\begin{array}{l}\text { müszorgalom }(<\text { Gewerbeflei } \beta ; 1831) \\
\text { ipar }(1828) \\
\text { iparos (< gewerbsam, , gewerbsam‘; 1835) } \\
\text { iparos (,Industrieller'; 1845) }\end{array}$ & $\begin{array}{l}\text { ipar } \\
\text { iparos }\end{array}$ \\
\hline Schreibstube ('Büro') & iroda $(1842) /$ irota $(1845)$ & iroda \\
\hline $\begin{array}{l}\text { Begünstigung } \\
\text { begünstigt } \\
\text { begünstigen }\end{array}$ & $\begin{array}{l}\text { kedvezmény }\left(, V^{\prime} \text { Vorteil‘ 1832; ,Begünstigung' 1845) }\right. \\
\text { kedvezményes }(1857) \\
\text { kedvezményez }(1890)\end{array}$ & \begin{tabular}{|l|} 
kedvezmény \\
kedvezményes \\
kedvezményez \\
\end{tabular} \\
\hline Bilanz & mérleg (,Waage‘ 1823; ,Bilanz‘ 1832) & \begin{tabular}{|l|} 
mérleg \\
*mérleg (,Waage")
\end{tabular} \\
\hline
\end{tabular}




\begin{tabular}{|l|l|l|}
\hline $\begin{array}{l}\text { ausstellen } \\
\text { Ausstellung }\end{array}$ & $\begin{array}{l}\text { kiállit }(1803) \\
\text { kiállitás }(1803)\end{array}$ & $\begin{array}{l}\text { kiállit } \\
\text { kiállitás }\end{array}$ \\
\hline Wechsel(als Wertpapier) & váltó (1832) & váltó \\
\hline
\end{tabular}

Tab. 1: Beispiele für Ergebnisse der fachsprachlichen Übersetzungen aus dem Deutschen ${ }^{2}$

* Es lebt in umgangssprachlicher Bedeutung weiter

\section{Reinigung des Fachwortschatzes}

Die Bestrebungen nach einer einheitlichen wirtschaftlichen und juristischen ungarischen Fachsprache wurden im dritten Drittel des 19. Jhs. immer mehr aufeinander abgestimmt. Diese Periode ist schon durch den bewussten Gebrauch und die bewusste Verbreitung von Termini geprägt. Es ist in erster Linie durch die Lehrbücher repräsentiert (vgl. Nyomárkay 2007:186). Einige Wörterbücher sind aber auch aus dieser Epoche von großer Bedeutung, z. B. das Werk von Gergely Czuczor und János Fogarasi, dessen Bände zwischen 1862 und 1874 erschienen (Czuczor-Fogarasi 1862-1874). Dieses Wörterbuch wird hier aber nicht nur wegen seiner Beliebtheit und Auswirkung vorgeführt. Es ist einerseits für die Arbeit an der Aufdeckung vom Wortschatz der Gemeinsprache vom 19. Jh. unentbehrlich, andererseits stellt es aber eine wichtige und zuverlässige Quelle für die fachsprachgeschichtlichen Forschungen dar, da János Fogarasi - dessen Werke auch bei der Arbeit am ,Gesetzwissenschaftlichen Sachwörterbuch' benutzt worden sind - als Wechselanwalt tätig war, so enthält das Wörterbuch auch eine bedeutende Anzahl an juristischen Termini. Neben den zahlreichen Terminivarianten stellt das Wörterbuch auch die fachsprachliche Synonymie und Polysemie dar.

Bedeutungswandel der übersetzten Termini - ein Beispiel: Unternehmer

In meiner sprachgeschichtlichen Forschung von Termini wurden 1050 ungarische Fachwörter aufgenommen und ihr Bedeutungswandel analysiert. Als Quelle habe ich ein ungarisch-deutsches Wörterbuch aus der Zeit der ungarischen Spracherneuerung, das ,Ujdon-uj magyar szavak tára' ... ${ }^{3}$ von Királyföldy (1854) gewählt. Für die gefundenen Termini habe ich dann die folgenden Wörterbücher untersucht:

1. CZUCZOR G. - FOGARASI J. (Hrsg.) (1862-1874): A magyar nyelv szótára. Pest: Emich. ${ }^{4}$

2. SZILY K. (Hrsg.) (1902-1908): A Magyar Nyelvújitás Szótára. Budapest: Hornyánszky. ${ }^{5}$

3. RÉVÉSZ, V. (1910): Jogi Müszótár. Bécs: Manz. ${ }^{6}$

4. (Ohne Autorangabe) (1913): Jogi Szótár. Budapest: Grill. ${ }^{7}$

5. BENKÖ L. (Hrsg.) (1967-1976): A magyar nyelv történeti-etimológiai szótára. Budapest: Akadémiai. ${ }^{8}$

6. BÁRCZI G. - ORSZÁGH L. (Hrsg.) (1959-1962): A magyar nyelv értelmezö szótára. Budapest: Akadémiai. ${ }^{9}$

\footnotetext{
${ }^{2}$ Hier sind nur die Termini als Beispiele aufgelistet, die auch heute Elemente des ungarischen Fachwortschatzes sind. Es gibt natürlich viel mehr, wie auch viele andere, die schon aus dem Fachwortschatz verwunden sind.

3 Deutsches Titelblatt: ,Lexikon der neuen ungarischen Wörter, enthaltend die in den Zeitungen, neuen Büchern, in den Wissenschaften und im mündlichen Verkehr vorkommenden modernen Ausdrücke; technische Benennungen und eine grosse Anzahl neugebildeter und wieder belebter ungarischer Wörter.' Der volle ungarische Titel ist im Literatur- und Quellenverzeichnis zu finden.

4 [Wörterbuch der ungarischen Sprache]

5 [Wörterbuch der ungarischen Spracherneuerung]

${ }^{6}$ [Juristisches Fachwörterbuch]

7 [Wörterbuch des Rechts]

${ }^{8}$ [Historisch-etymologisches Wörterbuch der ungarischen Sprache]

9 [Bedeutungswörterbuch der ungarischen Sprache]
} 


\subsection{Die Untersuchungsmethode}

Zuerst habe ich alle Wörter und Ausdrücke in der Quellenausgabe untersucht und die Termini mit den deutschen Äquivalenten aufgelistet. So wurden 1050 Termini ausgewählt. Diese habe ich dann in den oben aufgezählten Wörterbüchern nachgeschaut und die vollen Einträge in einer Tabelle vorgeführt, um ihre semantischen Entwicklungsprozesse in chronologischer Reihenfolge darstellen zu können.

Das erste Wörterbuch war schon oben erwähnt - dies enthält sowohl die umgangssprachlichen als auch die fachsprachlichen Bedeutungen der ausgewählten Termini. Das zweite Wörterbuch zeigt, ob diese ungarischen Termini als Folgen der ungarischen Spracherneuerung gelten, und wie diese geschöpft worden sind. Die Wörterbücher Nr. 3. und 4. zeigten, ob das Wort/der Ausdruck Anfang des 20. Jhs. schon bzw. noch ein Element der juristischen Fachsprache war. Das fünfte Wörterbuch zeigt die möglichen Wortetymologien und gibt uns wichtige Informationen über die Entstehungsumstände. Als letztes macht uns das sechste Wörterbuch damit bekannt, ob die untersuchten Termini mitten des 20. Jhs. im Ungarischen existierten und wie sich ihre Bedeutung verändert hat.

Am Ende soll hier ein Beispiel stehen, das die ständige Veränderung der Fachsprache zeigt, wobei die Rolle der Gemeinsprache in diesem Prozess gut zu beobachten ist.

Dieser Terminus ist der Unternehmer.

Das ungarische Äquivalent des Fachwortes entstand in den 1830er Jahren: als der Terminus merénylö. Etwa zu gleicher Zeit verbreitete sich das Wort merény in der Gemeinsprache, aber in zwei unterschiedlichen Bedeutungen: einerseits als ,Wagestück', andererseits als ,Attentat' .

Infolge der Bestrebung nach Monosemie in der Fachsprache verloren die Wörter merény und merénylö ihre fachsprachlichen Bedeutungen, fungierten also nicht mehr als Fachwörter.

In der Fachsprache wurden sie durch die Termini vállalkozás (für Unternehmung) und vállalkozó (für Unternehmer) abgelöst.

In der Gemeinsprache trennten sich die zwei Bedeutungen weiter: mer oder merészel und merész bedeuten ,wagen“ und ,wagemutig'; während merénylet, merénylö ,Attentat' und ,Attentäter' sind.

\section{Schluss}

Meine Forschung fokussiert aber nicht nur auf die Analyse der juristischen Fachwörterbücher, sondern auch auf die Anforderungen, die sowohl während der Kodifikation als auch während der juristischen Fachübersetzung beachtet werden sollen. Die Erwartungen beider Fachbereiche zeigen, dass sowohl die Kodifikatoren als auch die Fachübersetzer über solche wichtigen Fähigkeiten verfügen sollen wie z. B. die Formulierungsfähigkeit oder die Fähigkeit zum Gebrauch der Muttersprache auf höchstem Niveau. Auch diese sind in den Kursen für Fachübersetzer zu entwickeln, aber das ist schon das Thema eines anderen Beitrags.

\section{Literaturverzeichnis}

\section{Primärliteratur:}

BÁrCZI, G. - OrszÁGH L. (Hrsg.) (1959-1962): A magyar nyelv értelmezö szótára. Budapest.

BENKö, L. (Hrsg.) (1967-1976): A magyar nyelv történeti-etimológiai szótára. Budapest.

Czuczor, G. - Fogarasi J. (Hrsg.) (1862-1874): A magyar nyelv szótára. Pest.

KIRÁLYFÖLDI, E. (1854): Ujdon-uj magyar szavak tára, melly a hazai hirlapokban, uj magyar könyvekben, tudományos és közéletben előkerülö ujdon kifejezéseket, mü- és más legujabban alakitott vagy felélesztett szavakat német forditással foglalja magában. Pest.

Ponori Thewrewk, J. (1844): Werböczy István diák müszavai régi magyaritásokkal. Pozsony.

RÉvÉsz, V. (1910): Jogi Müszótár. Bécs.

SCHEdel, F. (Hrsg.) (1847): Törvénytudományi müszótár. Pest. 
Szily, K. (Hrsg.) (1902-1908): A Magyar Nyelvújítás Szótára. Budapest.

WerböczI, I. (1611): Decretvm Juris consuetudinarii Regni Hungariae et Transylvaniae... Mostan Deákul es Magyarul, Egy hasznos Regestromal egyetemben uyobban ki boczatatot cum gratia et privilegio. Debrecen.

(Ohne Autorangabe) (1913): Jogi Szótár. Budapest.

\section{Sekundärliteratur:}

Bakos, G. (1880): A magyar jogi münyelv kérdéséhez. (Zum Themenkreis der ungarischen Fachsprache). Budapest.

NyOMÁrKaY, I. (2004): Nyelveink múltja és jelene. (Vergangenheit und Gegenwart unserer Sprachen) Budapest.

NyOMÁRKAY, I. (2007): Nyelvújítások Közép-Európában a 19. században. (Spracherneuerungen im Mitteleuropa vom 19ten Jh.). In: Magyar Nyelvör 131/2, S. 185-195.

NyomÁrkay, I. (2010): Filológiánk tegnap és ma. (Unsere Philologie gestern und heute) Magyar Nyelvör. 134. évf. 2. szám, S. 129-140.

PókeCz Kovács, A. (2011): Schaffung der Handelsgesetze von 1840 durch die ungarische Nationalversammlung und deren Anwendung bis 1849. In: JURA 2011/1, S. 117-127.

PulszKy, F (1884) Életem és korom. (Mein Leben und Zeit). Budapest.

Terestyéni, F. (1955) Az állami élet nyelve. (Die Sprache der öffentlichen Hand) In: PAis, D. (Hrsg.) Nyelvünk a reformkorban. (Unsere Sprache in der Reformzeit). Budapest, S. 83-164. 